\title{
Overexpression of RPOTmp, an Arabidopsis RNA-polymerase with dual-targeting, influences plant growth and transcriptome
}

\author{
V.I. Tarasenko, A.I. Katyshev, I.V. Gorbenko*, V.I. Belkov, E.Yu. Garnik, Yu.M. Konstantinov, M.V. Koulintchenko \\ Siberian Institute of Plant Physiology and Biochemistry, Siberian Branch of the Russian Academy of Sciences, Irkutsk, Russia
}

DOI 10.18699/ICG-PlantGen2019-54

(c) Autors, 2019

* e-mail: gravov.chemistry@gmail.com

\begin{abstract}
In dicot plants, RPOTmp is a dual targeting protein, which is presumably involved in gene transcription in both mitochondria and chloroplasts. The Arabidopsis rpotmp knockout line has retarded growth and development, changed expression of mitochondrial genes and decreased activities of the mitochondrial respiratory complexes. However, the particular importance of RPOTmp as a part of the mitochondrial and plastid transcription machinery remains unclear. In the current study, we used RPOTmp overexpressor lines with changed enzyme targeting directing either into mitochondria or chloroplasts, to study the particular role of this RNA polymerase in organelle gene expression and its plausible involvement in retrograde regulation. We have shown that RPOTmp overexpression influences plant growth and development and leads to significant changes in transcriptome. Key words: Arabidopsis thaliana; RPOTmp; mitochondria; chloroplast; gene expression.
\end{abstract}

\section{Introduction}

In a number of dicotyledonous plants, including Arabidopsis, transcription of organellar genes is performed by three nuclearencoded RNA polymerases, RPOTm, RPOTmp, and RPOTp. RPOTm performs the main function in plant mitochondria; RPOTp, along with PEP, a plastid-encoded RNA polymerase, plays a similar role in plastids. RPOTmp is localized in mitochondria as well as in chloroplasts and presumably participates in the control of gene expression of both organelles. The role of RPOTmp in mitochondrial transcription is confirmed by many studies (Emanuel et al., 2006; Courtois et al., 2007; Kühn et al., 2009). The absence of RPOTmp in Arabidopsis leads to a decrease in the transcription of genes encoding the subunits of respiratory complexes I and IV. Despite the obvious, though not fully understood role of RPOTmp in mitochondrial transcription, the function of this NEP polymerase in dicotyledonous chloroplasts is still under discussion. Many genetic elements in plastids and mitochondria are regulated by different types of promoters, implying competing or cooperative transcription of genes by different types of enzymes. Transcription of the plastid genome of lower plants, including algae, with the possible exception of Physicomitrella, is carried out only by PEP. In contrast, the benefits of using phage-type NEP polymerase by angiosperms, and even two of them by eudicots, remain unknown.

Since the localization and activity of RPOTmp is associated with two organelles, very different in their functions and type of gene expression, elucidation of specific functions of this RNA polymerase in each of the organelles is of considerable interest. Earlier (Tarasenko et al., 2016) we obtained two genetic constructs in which the gene sequence of the catalytic part of the Arabidopsis rpot2 gene was combined with the sequence encoding the transit peptide of mitochondrial RNA polymerase (RPOTm) (mtTmp-pBI121) or with the sequence encoding the transit peptide of chloroplast RPOTp (ptTmp-pBI121) (Figure 1, $a$ and $b$ ). These genetic constructs were used for agrobacterial transformation of Arabidopsis inflorescences (Clough, Bent, 1998) of the wild type or of the rpotmp knockout line. The following variants of transgenic plants were obtained (Figure 1,c): wild-type plants expressing recombinant protein RPOTmp targeted (1) to mitochondria (Col-M) or (2) to chloroplasts (Col-P); plants of the rpotmp mutant line, expressing recombinant protein targeted (3) to mitochondria (Tmp-M) or (4) to chloroplasts (Tmp-P).

The aim of this study was to find out more detail about the role RPOTmp plays in the regulation of organelle transcription and possibly in retrograde regulation of organelle functions.

\section{Materials and methods}

\subsection{Plant material and growth conditions}

Wild-type Arabidopsis thaliana (L.) Heynh plants (Col-0) and mutant line GABI_286E07 from the collection of GABIKat insertion mutants (Rosso et al., 2003) were used. In the mutant line, a T-DNA insert is located in the first exon of the rpot 2 gene, preventing the formation of a functional RPOTmp protein (Kühn et al., 2009). After 3 days at $4{ }^{\circ} \mathrm{C}$, plants were grown in pots at $24^{\circ} \mathrm{C}$ day $/ 21^{\circ} \mathrm{C}$ night, a 16 -h photoperiod and irradiance of $150 \mu \mathrm{mol} \mathrm{m}^{-2} \mathrm{~s}^{-1}$ in a KBW 720 growth chamber (Binder, Germany). A mixture of compost and Vermiculite (1:1) was used as a substrate. At least 50 plants of each line were used to estimate growth characteristics.

\subsection{Microarray analysis}

Total RNA samples were extracted from rosette leaves of 12-day-old plants using a Qiagen RNeasy Plant Mini Kit (Qiagen). RNA samples were labeled with a Low Input Quick Amp Labeling Kit, two-color, (Agilent Technologies) and an Agilent RNA Spike-In Kit, two-color, (Agilent Technologies). The cRNAs samples of Col- 0 and transgenic lines were labeled with $\mathrm{Cy} 3$ and $\mathrm{Cy} 5$ fluorescent dyes, respectively. Microarray analysis was performed by hybridizing four arrays in a single Arabidopsis (V4) Gene Expression Microarray slide $(4 \times 44 \mathrm{~K}$; Agilent Technologies) with a mixture of the Cy3- and Cy5labeled cRNAs. After hybridization using a Gene Expression Hybridization Kit (Agilent Technologies), the microarray slide 
was scanned using a scanner model G2539A with scan control A.8.5.1 (Agilent Technologies). Data analysis was performed using Feature Extraction 10.10.1.1 (Agilent Technologies) and GeneSpring GX 12.6.1 (Agilent Technologies). The p-value of each gene was calculated by two-way ANOVA and corrected by the Benjamini and Hochberg false discovery rate (FDR) method. Differential expression between samples was considered to be statistically significant at $p<0.05$ and FDR $<0.1$.

\section{Results and discussion}

\subsection{Phenotypic characteristics of Arabidopsis RPOTmp overexpressor lines}

The seeds of several homozygous lines with overexpression of RNA polymerase RPOTmp (Col-M and Col-P) were planted on Phytagel and on soil. The rate of seed germination, growth and development, the appearance of plants of Col-M lines were similar to those of the wild-type plants (Figure 1, $d$ ). The Col-P lines showed some differences from the wild-type plants. One out of six Col-P lines studied, Col-P12, was characterized by somewhat accelerated growth on the solid medium and on the soil and by a significantly $(p<0.05)$ earlier onset of flowering compared to the wild-type line. In contrast, three lines, Col-P5, Col-P8, and Col-P18, had a tendency for slow growth. Delayed seed germination and growth were expressed to a much greater extent in one of the lines, Col-P5 (see Figure $1, d)$. Also, plants of this line showed a pronounced delay in flowering, demonstrating in this respect a similarity with the rpotmp mutant plants. In addition, it can be noted that the plants of this line had the yellowish color of the first pair of true leaves at the time of their formation (a unique feature, not marked in any of the other lines studied), increased leaf folding in mature rosettes, a more rounded and curled shape of the leaves. The latter features also resemble the phenotype of the rpotmp mutant plants with inactivated expression of RPOTmp.

\subsection{Transcriptome analysis of Arabidopsis RPOTmp overexpressor lines}

Using the DNA microarray method, we performed a genomewide analysis of changes in the level of nuclear, chloroplast and mitochondrial gene transcripts in rpotmp mutant lines, lines overexpressing RPOTmp with mitochondrial or chloroplast targeting (Col-M15, Col-M20, Col-5, Col-P12), and in lines with complementation of RPOTmp functions in mitochondria (Tmp-M3, Tmp-M11) or in chloroplasts (Tmp-P1), in comparison with the wild-type plants. Total RNA extracted from 12-day-old seedlings was used for the analysis. Three biological repeats of RNA preparations obtained from several independently grown plants were used for analysis. From the resulting dataset, the genes, the expression level of which was statistically significant $(p<0.05)$ and at least two-fold different from the expression level of the wild-type plants, were selected for analysis. For each of the lines studied, several hundred genes met these criteria, which indicates that either the absence of RPOTmp in both (the rpotmp mutant) or one (the Tmp-M and Tmp-P lines) cell compartment, or the overexpression of the RNA polymerase leads to a significant response at the transcriptome level. Remarkably, most of the genes with altered expression were nuclear ones, showing that the observed changes can be attributed to retrograde regulation, that is, being dependent on the changes occurring in the organelles.

From the obtained it was clear that the expression changes in the rpotmp knockout line were similar to a high degree to that observed in the Tmp-P1 line (Figure 2), indicating that RPOTmp targeting to chloroplasts does not lead to compensation of defects characteristic of the mutant. In Tmp-M3 and Tmp-M11 lines, the number of genes with altered expression is the lowest; in this respect, these lines are close to the Col-P12 line with RPOTmp overexpression in chloroplasts, but not to the Col-P5 line. The Col-M15 line transcriptome was enriched for genes with an increased level of expression, which stand out in a separate cluster (Figure 2).

In addition, genes encoded in chloroplast and mitochondrial genomes were analyzed. It is interesting to note that none of the chloroplast genes were represented among genes with significantly altered expression in eight lines studied, which also indicates against any significant role RPOTmp can play in the transcription of the chloroplast genome. In contrast, the expression level of a number of mitochondrial genes was significantly altered. The data obtained using DNA microchips confirmed a decrease in the expression in the rpotmp mutant for all mitochondrial genes (such as rps4, matR, cox1, nad1, nad5, nad4, nad6, ccb256) shown previously by Kühn et al. (2009). In addition, several mitochondrial genes whose transcript levels in the absence of RPOTmp either decrease (orf100c, orf106d, orf107f, orf109, orf143, orf153a, orf215a, orf313, orfFX) or increase (orf110b, orf107c, orf111b, orf139a, orf141, orf184, orf 240a) have been identified by our analysis for the first time.

\section{Conclusion}

Analysis of changes in the level of mitochondrial gene transcripts allows us to draw conclusions about the almost complete identity of expression changes in the rpotmp and Tmp-P1 lines, and, conversely, a significant degree of compensation for these changes in the lines with RPOTmp complementation in mitochondria (Tmp-M3, Tmp-M11). The expression analysis of the mitochondrial genes in plants with RPOTmp overexpression showed a somewhat surprising picture. While the content of the mitochondrial gene transcripts in the Col-P12 line was close to that in the wild-type plants (which was to be expected in the line overexpressing RPOTmp with chloroplast targeting), the other line, Col-P5, was very close in this respect to the rpotmp knockout line. This fact correlates well with the phenotypic appearance and the level of mitochondrial genome copy number of plants of this line (the data are not shown). Analysis of the level of mitochondrial transcripts in the lines overexpressing RPOTmp with mitochondrial targeting (ColM15, Col-M20) also showed significant differences between these lines. While the Col-M15 line was characterized by an increased level of most mitochondrial transcripts, the content of many transcripts in the Col-M20 line, in contrast, was reduced, and the character of the changes was similar to that of the rpotmp and Col-P5 lines. It is interesting to note that the phenotype of these plants did not differ significantly from the phenotype of the wild-type plants. 
A Structure of nuclear encoded organellar RNA-polymerases
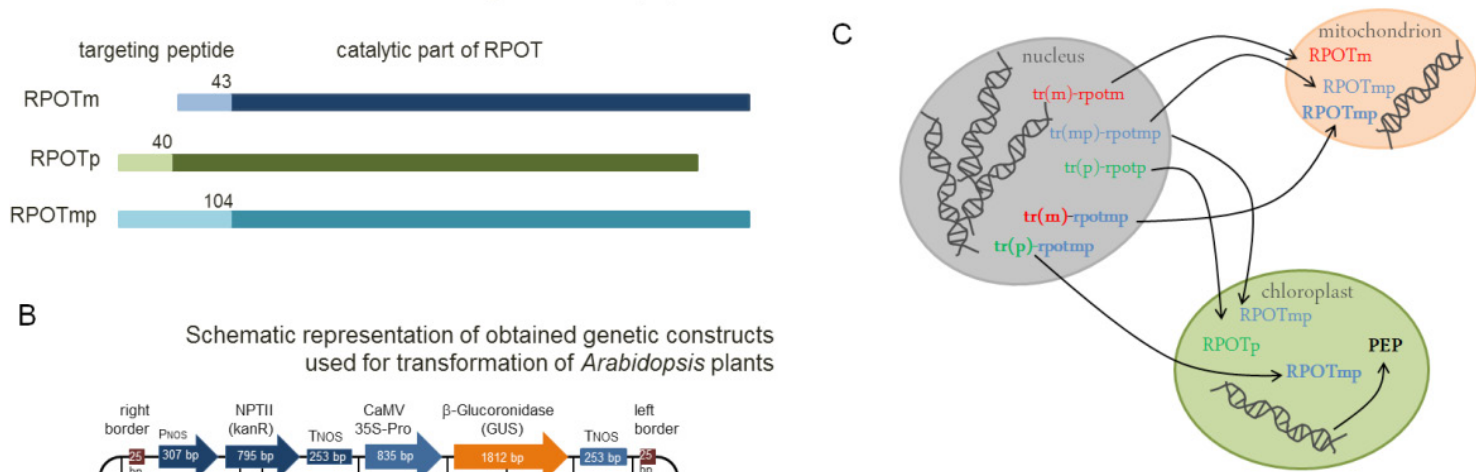

B

Schematic representation of obtained genetic constructs used for transformation of Arabidopsis plants

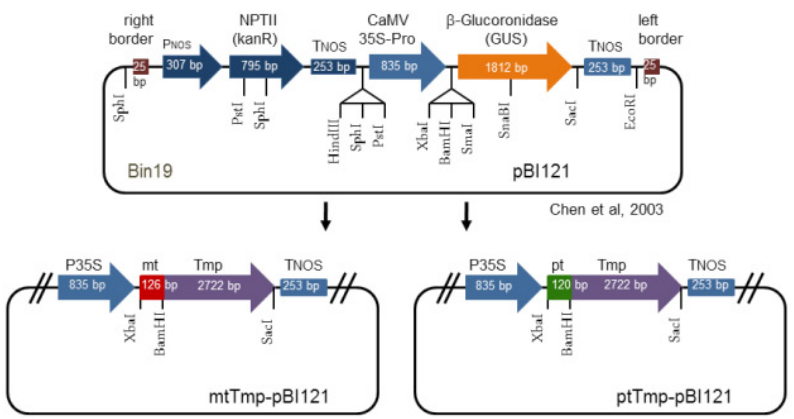

\section{D}
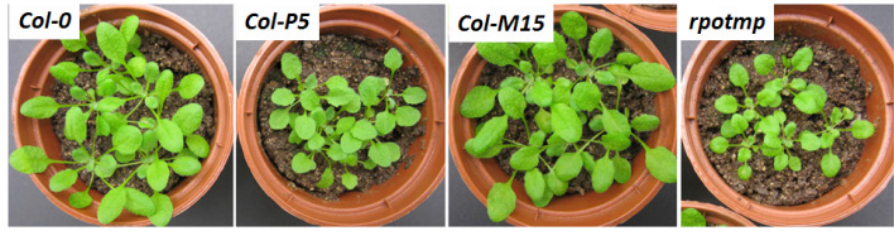

Figure 1. Transgenic Arabidopsis lines overexpressing RPOTmp with mitochondrial or chloroplast targeting. (a) Schematic representation of gene structures encoding three Arabidopsis RPOT polymerases. (b) Scheme of obtained genetic constructs (from Tarasenko et al., 2016). (c) Schematic representation of native and recombinant RPOTmp localization in cellular organelles. $\operatorname{tr}(m), \operatorname{tr}(p), \operatorname{tr}(m p)$ : sequences encoding transit peptides of the Arabidopsis rpotm, rpotp and rpotmp genes, respectively. (d) Appearance of Arabidopsis wild-type plants (Col-0), rpotmp mutant line and lines overexpressing RPOTmp with mitochondrial (Col-M15) or chloroplast (Col-P5) targeting.

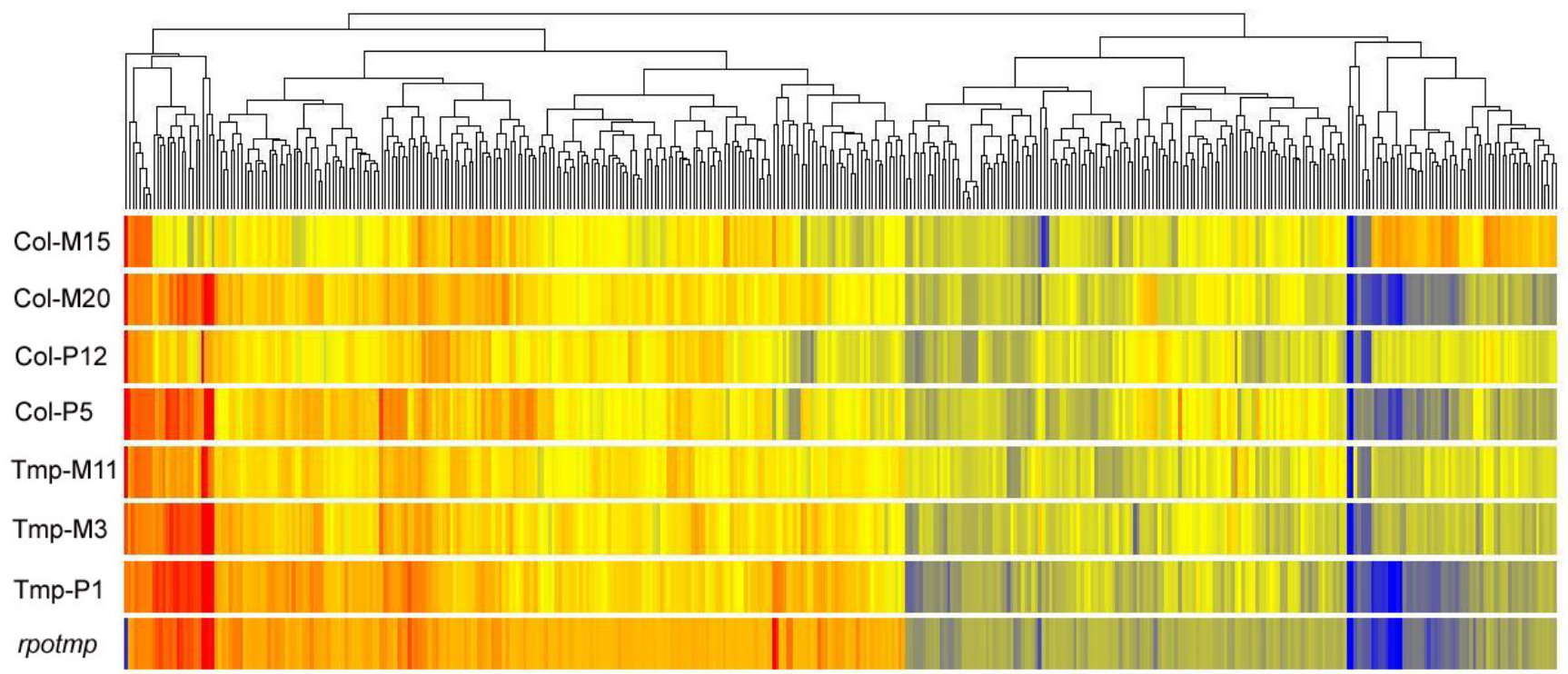

Figure 2. Dendrogram representing the clustering of genes differentially expressed in Arabidopsis lines overexpressing RPOTmp with mitochondrial or chloroplast targeting (Col-M, Col-P), in the rpotmp mutant line and in plants with RPOTmp complementation in mitochondria and chloroplasts (Tmp-M and Tmp-P). Red color means an increase in the level of gene expression compared to the level in the wild-type plants; blue represents a decrease. Genes whose expression levels differ at least two-fold from the level in wild-type plants $(p<0.05, n=3)$ are presented. 
It should also be noted that the increase in the expression of RPOTmp caused a change in the expression of many transcription factors belonging to certain classes (data not shown). Our task in subsequent studies will be to test the expression of selected genes encoding transcription factors of these potentially interesting classes.

Although we cannot, at the moment, make a definite conclusion about the reasons for the observed diversity of gene expression responses in the lines studied, it is obvious that the absence and increase in RPOTmp content produces different signals that lead to changes in expression in other cell compartments. At the same time, it seems that the change in the expression of the mitochondrial genome and/or the functional state of the mitochondria is one of the main sources and intermediaries in the transmission of this signal.

\section{References}

Chen P.Y., Wang C.K., Soong S.C., To K.Y. Complete sequence of the binary vector $\mathrm{pBI} 121$ and its application in cloning T-DNA insertion from transgenic plants. Mol. Breed. 2003;11:287-293.

Courtois F., Merendino L., Demarsy E., Mache R., Lerbs-Mache S. Phage-type RNA polymerase RPOTmp transcribes the rrn operon from the PC promoter at early developmental stages in Arabidopsis. Plant Physiol. 2007;145:712-21.
Emanuel C., von Groll U., Müller M., Börner T., Weihe A. Development- and tissue-specific expression of the RPOT gene family of Arabidopsis encoding mitochondrial and plastid RNA polymerases. Planta. 2006;223:998-1009.

Kühn K., Richter U., Meyer E., Delannoy E., de Longevialle A.F., O’Toole N., Börner T., Millar A., Small I., Whelan J. Phage-type RNA polymerase RPOTmp performs gene-specific transcription in mitochondria of Arabidopsis thaliana. Plant Cell. 2009;21:27622779.

Rosso M.G., Li Y., Strizhov N., Reiss B., Dekker K., Weisshaar B. An Arabidopsis thaliana T-DNA mutagenized population (GABI-Kat) for flanking sequence tag-based reverse genetics. Plant Mol. Biol. 2003;53:247-259.

Tarasenko V.I., Katyshev A.I., Yakovleva T.V., Garnik E.Y., Chernikova V.V., Konstantinov Y.M., Koulintchenko M. RPOTmp, an Arabidopsis RNA polymerase with dual targeting, plays an important role in mitochondria, but not in chloroplasts. J. Exp. Bot. 2016;67(19): 5657-5669. DOI 10.1093/jxb/erw327.

Acknowledgements. Equipment of the Bioanalitika Center for Collective Use, Siberian Institute of Plant Physiology and Biochemistry, Siberian Branch of the Russian Academy of Sciences, Irkutsk, was used in this study.

Conflict of interest. The authors declare no conflict of interest in financial or any other sphere. 